\title{
INTERVENCIÓN FONOAUDIOLÓGICA EN LA DISFAGIA NEUROGÉNICA JUSTIFICADA EN EVIDENCIAS
}

Armando Ivan Ramos Gutarra ${ }^{5}$

Actualmente son pocas las investigaciones científicas sobre la Disfagia Neurogénica en grupos homogéneos que permitan al terapeuta aplicar con certeza protocolos de evaluación y tratamiento. En el presente artículo se resumen las investigaciones revisadas en el proyecto ERABI (EvidenceBased Review of Rehabilitation of Moderate to Severe Acquired Brain Injuries), disponible en: http://www.abiebr.com

La disfagia se define como dificultad o molestias al deglutir. Se ha observado que el traumatismo craneoencefálico asociado a daño cortical y tronco encefálico focal y difuso puede alterar la capacidad deglutoria y dar lugar a la aparición de disfagia y aspiración ${ }^{11}$.

La disfagia después de una lesión cerebral adquirida (LCA) se ha atribuido a una disfunción muscular faríngea y a la falta de coordinación secundaria por la pérdida de control del sistema nervioso central. Los problemas de deglución más frecuentes en los pacientes con LCA comprenden tránsito oral prolongado (87,5\%), reflejo deglutorio diferido (87,5\%), acumulación en las valléculas $(62,5 \%)$ y acumulación en los senos piriformes (62,5\%), con presencia de aspiración en el 37,5\% de los pacientes con disfagia ${ }^{3}$.

La incidencia de disfagia en los pacientes que empiezan rehabilitación después de una LCA oscila entre el $25 \%$ y $78 \%$. Se ha demostrado que esta incidencia varía según la definición de disfagia utilizada y la situación del paciente en el momento del ingreso, estas diferencias permiten explicar las divergencias en los reportes de incidencia entre el $42 \%-65 \%$ en pacientes ingresados en una unidad de rehabilitación para LCA ${ }^{4,10,15,3,20}$, así como la naturleza de las observaciones clínicas; a saber:

1) La aspiración después de una LCA es del $30 \%$ al $50 \%$ en los pacientes con disfagia. Esto representa el 10\%-20\% de los pacientes ingresados para rehabilitación ${ }^{18,13,10,15}$. La incidencia de aspiración asintomática en los pacientes con LCA no está bien documentada. Estos casos pueden pasarse por alto si no se realizan estudios de Videofluoroscopía de la deglución ${ }^{9,6,12,7,18}$.

2) Los pacientes con puntuaciones FOIS (Functional Oral Intake Scale), GCS (Glasgow Coma Scale), RLAS (Rancho Los Amigos Scale) bajas tienen más probabilidades de contraer una neumonía durante la alimentación por sonda. El riesgo de contraer una neumonía es proporcional a la intensidad de la aspiración ${ }^{5}$.

3) La aspiración no puede diferenciarse de la penetración laríngea mediante una evaluación a la cabecera del paciente, resultando en un diagnóstico excesivo de aspiración y, en algunos casos, restricciones alimentarias innecesarias ${ }^{17}$.

5. Tecnólogo Médico especialista en Terapia de Lenguaje. Especialista en Motricidad Orofacial. Cirujano Den tista. Magister en Pedagogía y Didáctica Universitaria. Catedrático en la UNIFE - Universidad Femenina del Sagrado Corazón hasta el 2015. Director General de IRFMO - Instituto de Rehabilitación Formación en Motricidad Orofacial, Director Académico de CMOL - Comunidad de Motricidad Orofacial Latinoamerica na. Miembro de Operación Sonrisa - Perú, Miembro del comité editorial de REVMOF 
4) El riesgo de aspiración relacionada con disfagia es proporcional a la gravedad inicial del traumatismo craneal. Los antecedentes de traqueotomía o ventilación mecánica también pueden asociarse a un mayor riesgo de aspiración ${ }^{6}$.

5) Los estudios de Videofluoroscopía de la deglución, pueden emplearse para facilitar el manejo de la disfagia y la identificación de aspiración en la población con LCA. No hay datos científi cos concluyentes de que la Endoscopía de la deglución, sea más sensible que la Videofluoros copía de la deglución, para evaluar si un paciente presenta dificultad para deglutir o aspira ción después de un ictus. Aunque la Endoscopía de la deglución quizá sea menos invasiva y menos costosa se precisan más investigaciones para determinar su eficacia en la población con LCA/TCE ${ }^{1,8}$.

6) Por otro lado, hay datos científicos limitados que respaldan el uso de la Pulsioximetría para detectar aspiración en los pacientes que han sufrido un ictus. La Pulsioximetría no parece ser una prueba tan sensible como la Videofluoroscopía para determinar la aspiración después de un ictus. Se necesitan más investigaciones en las poblaciones con ictus y LCA 2, 14, 16, 17,19.

\section{REFERENCIAS BIBLIOGRÁFICAS}

1. Aviv, J. E., Kaplan, S. T., Thomson, J. E., Spitzer, J., Diamond, B., \& Close, L. G. (2000). The safety of flexible endoscopic evaluation of swallowing with sensory testing (FEESST): an analysis of 500 consecutive evaluations. Dysphagia, 15, 39-44.

2. Collins, M. J. \& Bakheit, A. M. (1997). Does pulse oximetry reliably detect aspiration in dysphagic stroke patients? Stroke, 28, 1773-1775.

3. Field, L. H. \& Weiss, C. J. (1989). Dysphagia with head injury. Brain Inj, 3, 19-26.

4. Halper, A. S., Cherney, L. R., Cichowski, K., \& Zhang, M. (1999). Dysphagia after head trauma: the effect of cognitive-communicative impairments on functional outcomes. J Head Trauma Rehabil, 14, 486-496.

5. Hansen, T. S., Larsen, K., \& Engberg, A. W. (2008). The association of functional oral intake and pneumonia in patients with severe traumatic brain injury. Arch Phys Med Rehabil, 89, 2114-2120.

6. Horner, J. \& Massey, E. W. (1988). Silent aspiration following stroke. Neurology, 38, 317-319.

7. Lazarus, C. \& Logemann, J. A. (1987). Swallowing disorders in closed head trauma patients. Arch Phys Med Rehabil, 68, 79-84.

8. Leder, S. B. \& Espinosa, J. F. (2002). Aspiration risk after acute stroke: comparison of clinical examination and fiberoptic endoscopic evaluation of swallowing. Dysphagia, 17, 214-218.

9. Linden, P. \& Siebens, A. A. (1983). Dysphagia: predicting laryngeal penetration. Arch Phys Med Rehabil, 64, 281-284

10. Mackay, L. E., Morgan, A. S., \& Bernstein, B. A. (1999b). Swallowing disorders in severe brain injury: risk factors affecting return to oral intake. Arch.Phys.Med.Rehabil, 80, 365-371.

11. Morgan, A. T. \& Ward, E. C. (2001). Swallowing: neuroanatomical and physiological framework. In B.E.Murdoch \& D. G. Theodoros (Eds.), Traumatic brain injury: associated speech, language, and swallowing disorders (pp. 313-329). San Diego: Singular Publishing Group.

12. Muller-Lissner, S. A., Fimmel, C. J., Will, N., Muller-Duysing, W., Heinzel, F., \& Blum, A. L. (1982). Effect of gastric and transpyloric tubes on gastric emptying and duodenogastric reflux. Gastroenterology, 83, 1276-1279.

13. O'Neil-Pirozzi, T. M., Lisiecki, D. J., Jack, M. K., Connors, J. J., \& Milliner, M. P. (2003). Simultaneous modified barium swallow and blue dye tests: a determination of the accuracy of blue dye test aspiration findings. Dysphagia, 18, 32-38.

14. Sellars, C., Dunnet, C., \& Carter, R. (1998). A preliminary comparison of videofluoroscopy of swallow and pulse oximetry in the identification of aspiration in dysphagic patients. Dysphagia, 13, 82-86. 
15. Schurret, M. J., Ebner, K. A., Maser, A. L., Sperling, K. B., Helgerson, R. B., \& Harms, B. (1999). Formal swallowing evaluation and therapy after traumatic brain injury improves dysphagia. outcomes. J Trauma, 46, 817-821

16. Sherman, B., Nisenboum, J. M., Jesberger, B. L., Morrow, C. A., \& Jesberger, J. A. (1999). Assessment of dysphagia with the use of pulse oximetry. Dysphagia, 14, 152-156.

17. Smith, H. A., Lee, S. H., O'Neill, P. A., \& Connolly, M. J. (2000). The combination of bedside swallowing assessment and oxygen saturation monitoring of swallowing in acute stroke: a safe and humane screening tool. Age Ageing, 29, 495-499.

18. Terre, R. \& Mearin, F. (2009). Evolution of tracheal aspiration in severe traumatic brain injuryrelated oropharyngeal dysphagia: 1-year longitudinal follow-up study. Neurogastroenterol. Motil., 21, 361-369.

19. Wang, T. G., Chang, Y. C., Chen, S. Y., \& Hsiao, T. Y. (2005). Pulse oximetry does not reliably detect aspiration on videofluoroscopic swallowing study. Arch Phys Med

20. Winstein, C. J. (1983). Neurogenic dysphagia. Frequency, progression, and outcome in adults following head injury. Phys.Ther., 63, 1992-1997. 